\title{
Damage Prediction Model of High Pressure Water Jet Impinging Coal Rock Based on PSO-BPNN
}

\author{
Jialiang Liu ${ }^{12}$ \\ Chongqing Key Laboratory of Traffic \& Transportation, Chongqing Jiaotong University, Chongqing, \\ 400074, China \\ College of Civil Engineering, Chongqing Jiaotong University, Chongqing Jiaotong University, \\ Chongqing, 400074, China \\ National and local joint engineering laboratory of traffic civil engineering materials, Chongqing \\ Jiaotong University, Chongqing 400074, China \\ E-mail: Iiuacademicohotmail.com
}

\author{
Jianping Gao ${ }^{a}$, Mengjin Wang ${ }^{b}$ \\ College of Civil Engineering, Chongqing Jiaotong University, Chongqing Jiaotong University, \\ Chongqing, 400074, China \\ E-mail: ${ }^{a}$ jianping-gao@163. com; ${ }^{b}$ wangmengjin_lemon@163.com
}

\section{Dazhi Sun}

Department of Civil \& Architectural Engineering, Texas A\&M University-Kingsville, Texas 78363-8202, USA

E-mail: dazhi.sunetamuk.edu

In order to study the damage in coal rock under high pressure water jet, which is closely related to the efficiency of coalbed gas drainage, a three-dimensional damage numerical model is established. Based on the simulation results and PSO-BNPP (particle swarm optimization, PSO and backpropagation neural network, $B P N N$ ) method, a damage prediction model of high pressure water jet impacting coal rock was established. The results show that the mean absolute error, the mean absolute percentage error of the model are 1.23 and $7.45 \%$ respectively, and the actual and the predicted values show significant positive correlation. In this sense, the model can describe the relationship between the coal rock damage and the water jet parameters well.

CENet 2017

22-23 July, 2017

Shanghai, China

${ }^{1}$ This work is supported by the National Natural Science Foundation of China (NSFC) (No.:51608082 and 51508065), the Science and Technology Foundation of Chongqing Education Committee (No. KJ1500509), and The Applied Basic Research Foundation of Chongqing Science and Technology Committee (No. cstc2016jcyjA0374).

${ }^{2}$ Correspongding Author

(C) Copyright owned by the author(s) under the terms of the Creative Commons 


\section{Introduction}

As the unconventional resource, the coalbed gas is clean, high-quality and valuable, but the drainage is very difficult and complex. The high-pressure water jet technology is applicable in coalbed gas mining because of such significant advantages as no heat effect, high flexibility and high surface quality, etc. [1]. At present, it has been applied to many mining areas successfully, for example, Huainan, Jiaozuo, and Xinmi in China, especially for the coal seams with deep buried, low permeability and gas outburst [2].

Under the high-pressure water jet impacting, the damage in coal rock is the key factor of drainage efficiency because it will affect the porosity directly; therefore, it is necessary to establish a damage prediction model in coal rock under high pressure water jet impacting. As the high pressure water jet drilling is a nonlinear course involving many factors including water jet parameters, coal rock structure and fluid-solid coupling, the robustness, scalability, convergence of traditional nonlinear fitting theories and artificial intelligence prediction methods, cannot fulfill the requirements [3]. As a result, on the basis of PSO model (Particle Swarm Optimization), this paper improves the $B P N N$ (back-propagation neural network) model and establishes a damage prediction model in coal rock under high pressure water jet impacting, which will be helpful to improve the application of high pressure water jet technology in coalbed drainage.

\section{Numerical Model}

\subsection{Governing Equations}

$$
\begin{gathered}
\delta \rho / \delta t=-\rho \delta v_{i} / \delta x_{i}-w_{i} \delta \rho / \delta x_{i}(i, j=1,2,3) \\
v \delta v_{i} / \delta t=\sigma_{i j, j}+\rho b_{i}-\rho w_{i} \delta x_{j} / \delta x_{i} \\
\rho \delta E / \delta t=\sigma_{i j} v_{i, j}+\rho b_{i} v_{i}-\rho w_{i} \delta E / \delta x_{j}
\end{gathered}
$$

Where $\rho$ is the material density, $v_{\mathrm{i}}$ is the material velocity, $w_{\mathrm{i}}$ is the relative velocity between material velocity and mesh, $\sigma_{\mathrm{ij}}$ is Cauchy stress, $b_{\mathrm{i}}$ is the body force and $E$ is the energy [4-5].

The constitute equations for coal rock, water jet and air are written as follows:

$$
\begin{gathered}
\sigma^{*}=\left(A(1-D)+B P^{* N}\right)\left(1+C \ln \varepsilon^{*}\right) \\
P=a_{1} \mu+a_{2} \mu^{2}+a_{3} \mu^{3}+\left(b_{0} \mu+b_{1} \mu^{2}+b_{2} \mu^{3}\right) E \\
P=c_{0}+c_{1} \mu+c_{2} \mu^{2}+c_{3} \mu^{3}+\left(c_{4}+c_{5}+c_{6} \mu\right) E
\end{gathered}
$$

Where $\sigma^{*}$ is the normalized equivalent stress, $A$ is the cohesion parameter, $B$ is the pressure hardening coefficient, $C$ is the strain-rate sensitivity coefficient, $N$ is pressure hardening exponent, $P^{*}$ is the normalized pressure, is the normalized strain rate. Damage $(D)$ is accumulated from both the equivalent plastic strain increment and the equivalent plastic volumetric strain increment, which is expressed as:

$$
D=\frac{\sum \Delta \varepsilon_{P}+\Delta \mu_{P}}{\varepsilon_{p}^{f}+\mu_{p}^{f}}
$$


Where $\Delta \varepsilon_{\mathrm{p}}$ and $\Delta \mu_{\mathrm{p}}$ are the equivalent plastic strain and the equivalent plastic volumetric strain respectively, $f(p)=\varepsilon_{\mathrm{p}}^{f}+\mu_{\mathrm{p}}^{f}=D_{1}\left(P^{*}+T^{*}\right)^{\mathrm{D} 2}$ is the material fracture plastic strain under actual pressure, $D_{1}$ and $D_{2}$ are the material constant. $T^{*}$ is the normalized hydrostatic tension [6-7].

The main mechanical parameters of coal rock are shown in Table 1.

\begin{tabular}{llllll}
\hline$\rho / g \cdot \mathrm{cm}^{-3}$ & $\mathrm{G} / \mathrm{GPa}$ & $f^{\prime}{ }^{\prime} / \mathrm{GPa}$ & $T^{*} / \mathrm{MPa}$ & $P_{\text {crush }} / \mathrm{GPa}$ & $\mu_{\text {crush }}$ \\
\hline 1.4 & 5.0 & 0.02 & 0.2 & 4 & 0.001 \\
\hline
\end{tabular}

Table 1: Main Mechanical Parameters of Coal Rock

\subsection{Geometrical Model and Boundary Condition}

A quarter model is simulated only due to the symmetry of the model. The whole computational area is $50 \mathrm{~mm} \times 50 \mathrm{~mm} \times 50 \mathrm{~mm}$, the coal rock $50 \mathrm{~mm} \times 50 \mathrm{~mm} \times 30 \mathrm{~mm}$, water jet $1.8 \mathrm{~mm}$ (diameter) $\times 20 \mathrm{~mm}$ (height), $50 \mathrm{~mm} \times 50 \mathrm{~mm} \times 20 \mathrm{~mm}$ for air. It sets up and down surfaces and side surfaces as the non-reflecting boundary, and $Y=0$ in $X O Z$ coordinate plane, $X=0$ in $Y O Z$ coordinate plane[7], as shown in Figure 1.

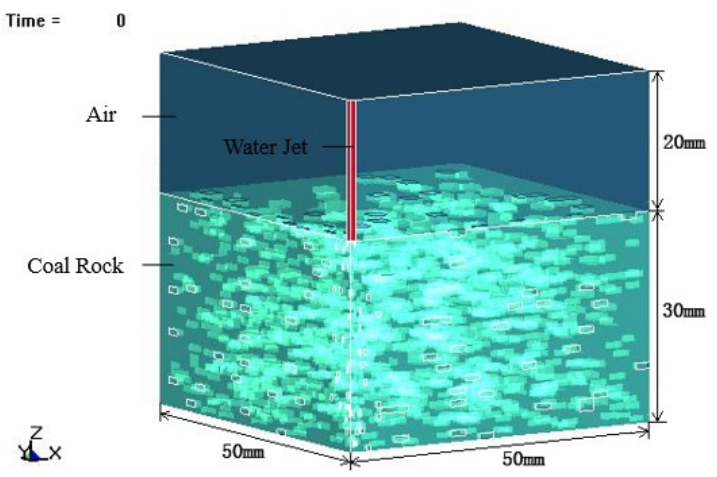

Figure 1: Overall Geometrical Model

\section{PSO-BPNN Neural Network}

\subsection{BPNN Model}

The $B P N N$ model propagates error reversely in the multi-layer network, and modifies the weight $\theta_{\mathrm{j}}$ and the threshold $\theta_{\mathrm{k}}$ by using the gradient descent algorithm [8]. The basic algorithm of $B P N N$ model includes the forward propagation of the input signals and the backward propagation of the error signals. In this paper, it selects the pump pressure $P$, the target distance $L$, the number of cuts $n$ and the traverse speed $v$ as the nerve nodes of the input layer, the damage $D$ as the output layer. The $B P N N$ topological structure is shown in Figure 2.

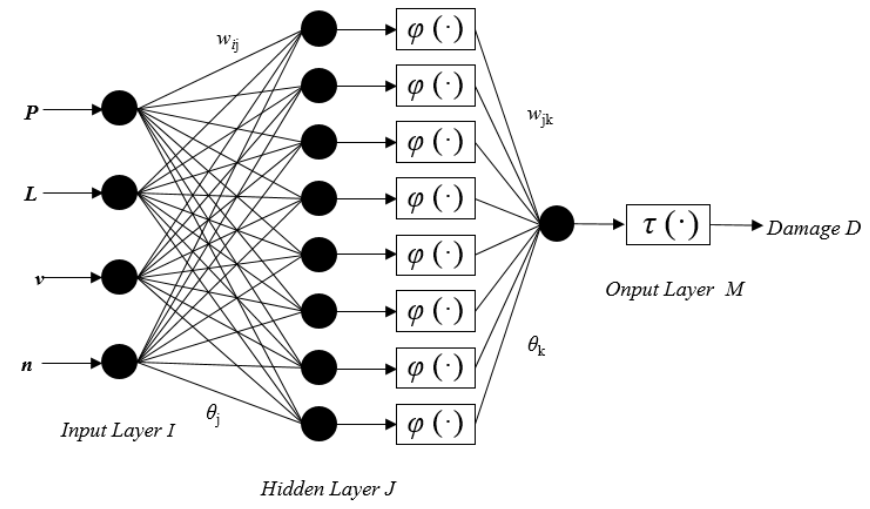

Figure 2: The BPNN Topological Structure of Damage in Coal Rock under High Pressure Water Jet Impacting 


\subsection{PSO-BPNN Prediction Model}

The initial weight and threshold of the BPNN model are optimized based on the PSO. To improve the local search ability of particles, this paper used larger inertia weight in the early stage and smaller inertia weight in the later stage [8]. The basic parameters of the PSO algorithm were set as follows: the population size $n=30$, the learning factor, the particle range was 0 , the range of particle position and velocity was $[-5,5]$ and $[-1,1]$, the maximum number of iterations was 100 and the maximum limit speed was 1 . The optimal process of POS-BPNN is shown in Figure 3.

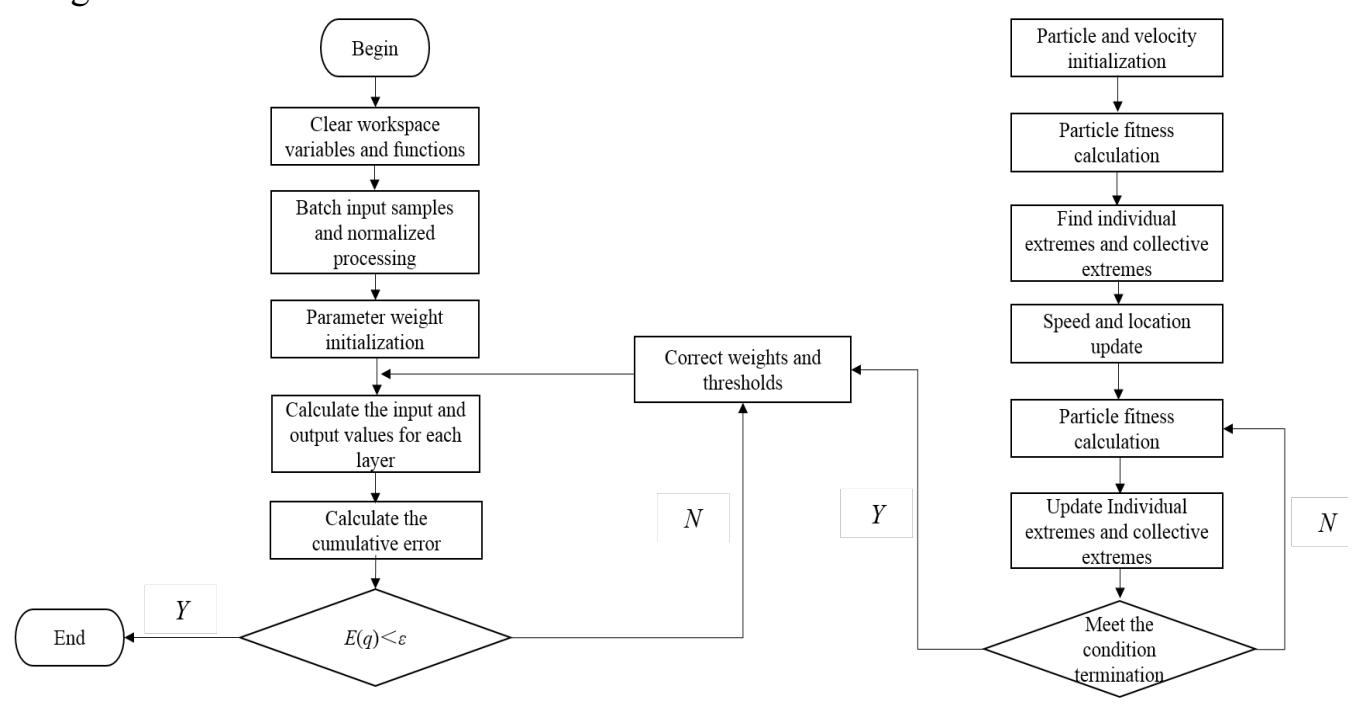

Figure 3: The Algorithm Flowchart of the POS-BPNN

\section{Results and Analysis}

Twenty-four sets of numerical simulation data are selected as the training samples. Figure 4 shows the mean square error of coal rock damage prediction model under water jet impacting, and we can see that it achieves the target error $(\varepsilon=0.0001)$ when the iteration times is 19 and the learning speed is very fast. Figure 5 shows the regression curve of damage prediction model in the coal rock under water jet, and it can be seen that the fitting coefficient $R=0.99381$, and it is quite closely to 1 . As a result, the model can fit the samples accurately.

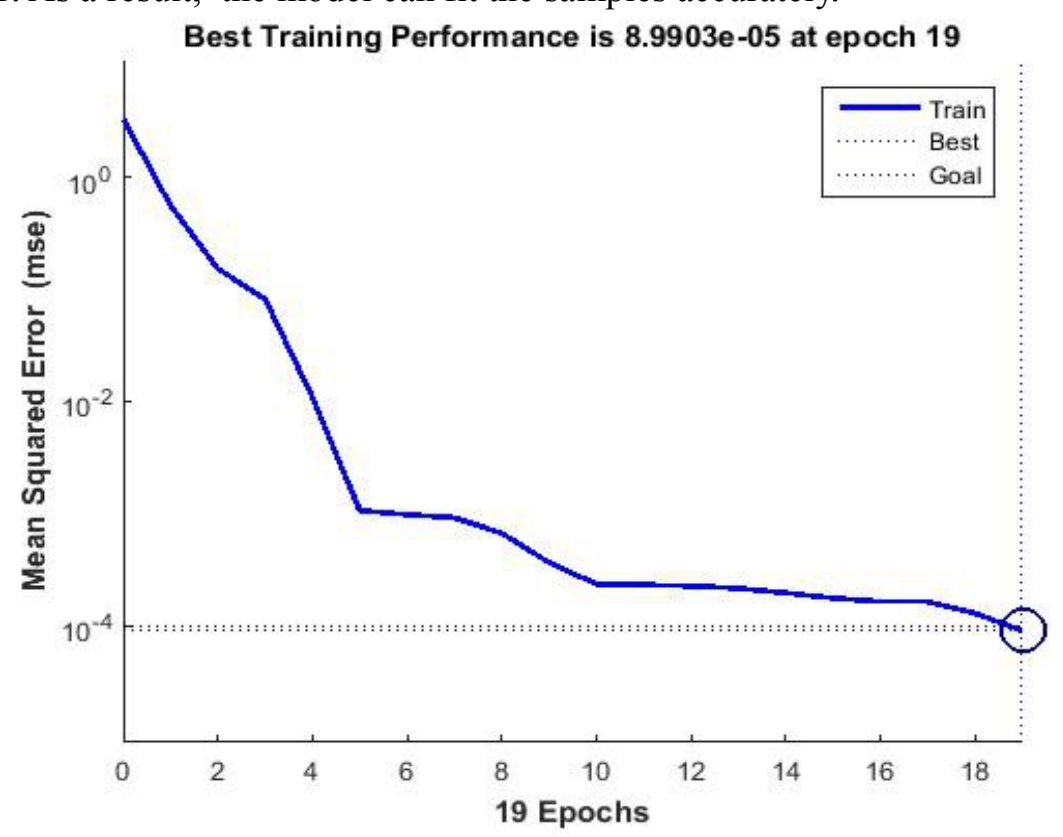

Figure 4: The Mean Square Error of Water Jet Impacting Coal Rock Damage Prediction Model 


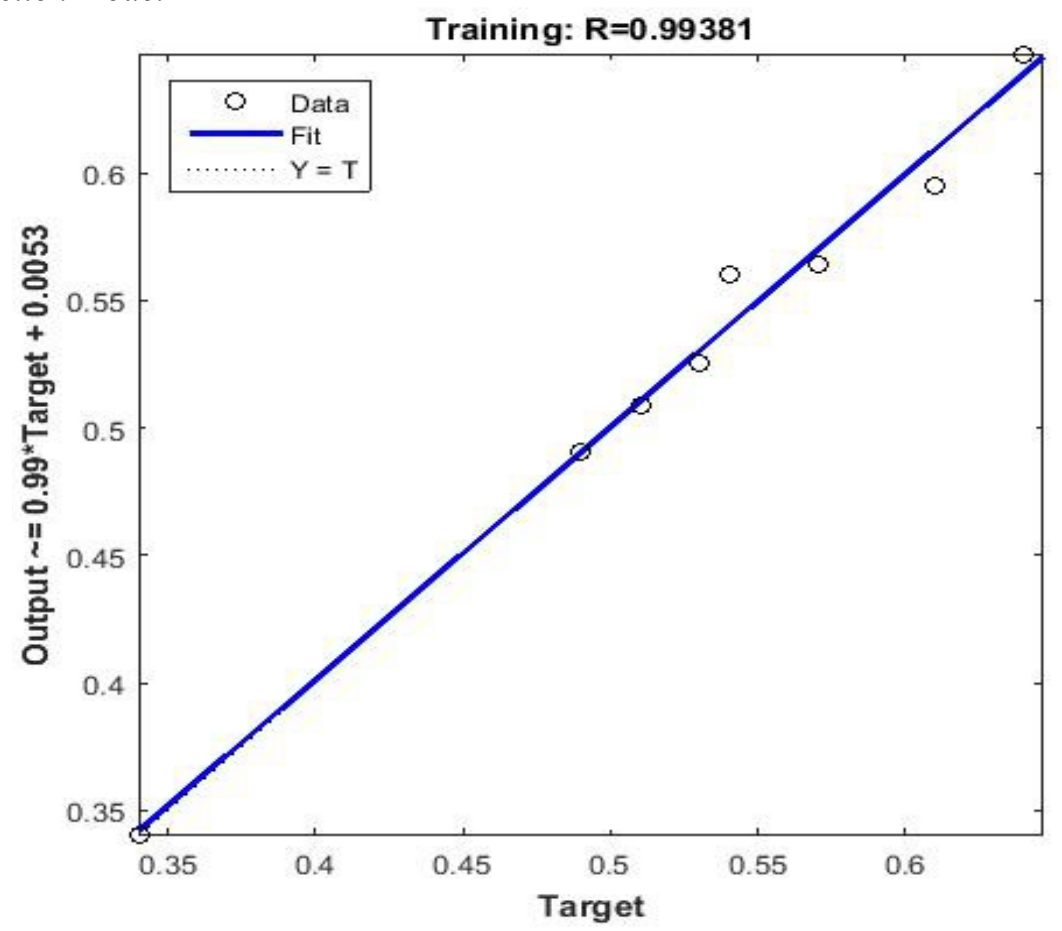

Figure 5: The Regression Curve of Damage Prediction Model in Coal Rock under Water Jet Impacting

Then the paper chooses another eight sets of numerical simulation data to verify the established prediction model. Based on the comparison betweenthe prediction results and the actual values, the average absolute error $(M A E)$ of the model is 1.23 and the average absolute percentage error (MAPE) is $7.45 \%$. The results show the prediction model of the damage in coal rock under high pressure water jet features good global convergence, learning efficiency and prediction accuracy. Therefore, it can describe the relationship between the damage in coal rock under high pressure water jet impacting and the water jet parameters well.

\section{Conclusion}

Based on the established three-dimensional damage numerical model and the PSO-BNPP method, a damage prediction model of high pressure water jet impacting coal rock is thereby built. The results show that the average absolute error $(M A E)$ and the average absolute percentage error $(M A P E)$ of the prediction model are both within a reasonable range. The prediction model shows a good prediction precision of the damage in coal rock by the high pressure water jet impacting.

\section{References}

[1] Yong Liu, An He, Jianping Wei, et al. Plugging factor and new plugging method to hydraulic relieving stress [J]. Journal of China Coal Society. 2016, 41(8):1963-1967(In Chinese).

[2] Yanzeng Li. Study of application of guide slot directional hydraulic pressurized penetration and permeability improved technology [J]. Coal Science and Technology. 2016, 4(4):50-54(In Chinese).

[3] Liming Xu, Qing Wang, Jianping Chen, et al. Forecasted the average velocity of debris flow based on BP neural network [J]. Journal of Jilin University. 2013, 43(1):186-191(In Chinese).

[4] Kai Wang, Bo Li, Jianping Wei, et al. After hydraulic punching drilling permeability change rule of around the seam [J]. Journal of Mining \& Safety Engineering. 2013, 30(5):778-784(In Chinese).

[5] Gadala M.S., Recent Trends in ALE Formulation and its Applications in Solid Mechanics [J], Computer Methods in Applied Mechanics and Engineering, 2004, 193, 4275-4275. 
[6] Yitian Wang, Jiazhong Zhang, An Improved ALE and CBS-based Finite Element Algorithm for Analyzing Flows around Forced Oscillating Bodies[J], Finite Elements in Analysis and Design, 2011, 47, 1058-1065 (In Chinese).

[7] Jialiang Liu, Hu Si. Gas Disaster Controlling Based on High Pressure Water Jet [J]. Disaster Advances, 2012, 5(4):1618-1625(In Chinese).

[8] Xiao Chen, Hongying Wang, Dandan Kong, et al. Quality prediction model of pellet feed basing on BP network using PSO parameters optimization method [J]. Transactions of the Chinese Society of Agricultural Engineering, 2016, 32(14):306-314(In Chinese). 